\title{
Probability in relativistic quantum mechanics and foliation of spacetime
}

\author{
Hrvoje Nikolić \\ Theoretical Physics Division, Rudjer Bošković Institute, \\ P.O.B. 180, HR-10002 Zagreb, Croatia \\ e-mail: hrvoje@thphys.irb.hr
}

February 5, 2018

\begin{abstract}
The conserved probability densities (attributed to the conserved currents derived from relativistic wave equations) should be non-negative and the integral of them over an entire hypersurface should be equal to one. To satisfy these requirements in a covariant manner, the foliation of spacetime must be such that each integral curve of the current crosses each hypersurface of the foliation once and only once. In some cases, it is necessary to use hypersurfaces that are not spacelike everywhere. The generalization to the many-particle case is also possible.
\end{abstract}

PACS numbers: 03.65.Pm, 03.65.Ta

Keywords: Relativistic quantum mechanics; probability density; foliation of spacetime.

\section{Introduction}

Finding a consistent probabilistic interpretation in the configuration space of relativistic quantum mechanics (QM) is a long-standing problem (see, e.g., [1, 2]). The simplest example leading to this problem is the Klein-Gordon equation (with the units $\hbar=c=1$ and the signature $(+,-,-,-))$

$$
\left(\partial^{\mu} \partial_{\mu}+m^{2}\right) \psi(x)=0
$$

The quantity $|\psi(x)|^{2}$ cannot be interpreted as the probability density because then the total probability $\int d^{3} x|\psi|^{2}$ would not be conserved in time. A better candidate for the probability density is the time component $j_{0}$ of the conserved current

$$
j_{\mu}=i \psi * \stackrel{\leftrightarrow}{\partial}_{\mu} \psi
$$

(where $a \overleftrightarrow{\partial}_{\mu} b \equiv a \partial_{\mu} b-b \partial_{\mu} a$ ), but the problem is that $j_{0}$ may be negative on some regions of spacetime, even if $\psi$ is a superposition of positive-frequency plane waves only. 
The Dirac equation of a single particle does not suffer from this problem, but a manyparticle generalization of the Dirac equation leads to a similar problem [3]. The usual solution of the problem is second quantization (see, e.g., [4, 2]), which postulates that $\psi$ is not a wave function determining probabilities, but an observable (called field) described by quantum field theory (QFT). Unfortunately, QFT is only a partial solution of the problem [5], because the axioms of QFT do not incorporate nor explain the probabilistic interpretation of $\psi$ in the nonrelativistic limit, despite the fact that the probabilistic interpretation of $\psi$ in the nonrelativistic limit is in agreement with experiments. Finding a consistent relativistic position-operator could also solve the problem, but it seems that a hermitian position-operator cannot be constructed in a covariant way [6, 7].

In this paper we propose a novel, Lorentz covariant, solution of the problem of probabilistic interpretation of relativistic QM. The main technical ingredient is the particle current, which can be introduced either as a QFT operator [8, 9, 10, 11, or a c-number quantity calculated from the wave function attributed to a QFT state [12, 13]. For simplicity, in this paper we study free particles, but we note that the particle current can be introduced even when the interactions with classical or quantum fields (that cause particle creation and destruction) are present [9, 10, 11, 12, 13. The main conceptual ingredient is the observation that, despite common practice, there is no a priori reason why the hypersurface on which the probability is defined should be spacelike everywhere. Indeed, such hypersurfaces that are not spacelike everywhere may appear in some variants of the many-fingered time formulation of QFT [14, 15, 16] and in the formulation of QFT based on the covariant canonical De Donder-Weyl formalism [17]. In this paper we show that hypersurfaces that are not spacelike everywhere naturally emerge from the requirement that the conserved c-number valued particle current should describe a probability density on a hypersurface.

\section{Particle current}

For example, consider a hermitian (uncharged!) scalar field operator $\hat{\phi}(x)$ that satisfies the Klein-Gordon equation (11). Denoting by $|0\rangle$ and $|1\rangle$ the Lorentz-invariant QFT states corresponding to the vacuum and a 1-particle state, respectively, the corresponding wave function [2]

$$
\psi(x)=\langle 0|\hat{\phi}(x)| 1\rangle
$$

is a superposition of positive-frequency plane waves only. The corresponding c-number valued particle current is given by (2) [12]. Since $\psi$, just like $\hat{\phi}$, satisfies (11), it follows that the current is conserved:

$$
\partial_{\mu} j^{\mu}=0 .
$$

As another example, consider the electromagnetic field operator $\hat{A}^{\alpha}(x)$ quantized using the covariant Gupta-Bleuler quantization [2]. In this case, the 1-photon wave function is

$$
\psi^{\alpha}(x)=\left\langle 0\left|\hat{A}^{\alpha}(x)\right| 1\right\rangle,
$$

while the particle current is

$$
j_{\mu}=-i \psi_{\alpha}^{*} \stackrel{\leftrightarrow}{\partial_{\mu}} \psi^{\alpha}
$$


The photon wave function satisfies $\partial^{\mu} \partial_{\mu} \psi^{\alpha}=0$ and the current (6) satisfies the conservation equation (44). For other examples of particle currents and their difference with respect to more familiar charge currents, see [9, 10, 11, 12, 13].

\section{$3 \quad$ Probability density and integral curves}

The currents defined as above have the property

$$
\int_{\Sigma} d S^{\mu} j_{\mu}=1
$$

where $\Sigma$ is an arbitrary 3-dimensional spacelike hypersurface and

$$
d S^{\mu}=d^{3} x\left|g^{(3)}\right|^{1 / 2} n^{\mu}
$$

is the covariant measure of the 3 -volume on $\Sigma$. Here $n^{\mu}$ is the unit future-oriented vector normal to $\Sigma$, while $g^{(3)}$ is the determinant of the induced metric on $\Sigma$. The crucial consequence of (4) is that (7) does not depend on the choice of the spacelike hypersurface $\Sigma$. Owing to this fact, one is tempted to interpret the scalar density

$$
\tilde{j} \equiv\left|g^{(3)}\right|^{1 / 2} n^{\mu} j_{\mu}
$$

as the probability density $\tilde{p}$ on $\Sigma$. (The tilde above a quantity denotes that this quantity does not transform as a tensor, but rather as a tensor density.) However, the probability density must satisfy the positivity requirement $\tilde{p} \geq 0$. Does $\tilde{j}$ satisfy the positivity requirement?

First, consider the case in which $j^{\mu}$ is timelike and future-oriented everywhere. In this case, $\tilde{j} \geq 0$, so it is fully consistent to write $\tilde{p}=\tilde{j}$. The probability of finding the particle on some finite 3 -dimensional region $\sigma \subset \Sigma$ is $P_{\sigma}=\int_{\sigma} d^{3} x \tilde{p}$. The probability $P_{\sigma}$ does not depend on $\sigma$ that belongs to a family of $\sigma$ 's constructed as follows (see Fig. 1): The vector field $j^{\mu}(x)$ defines the congruence of integral curves, such that, at each point $x$, the vector $j^{\mu}$ is tangential to the curve. The integral curves $x^{\mu}(s)$ can be parametrized such that

$$
\frac{d x^{\mu}}{d s}=j^{\mu}
$$

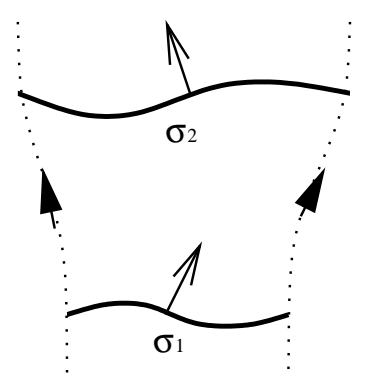

Figure 1: Spacelike regions $\sigma_{1}$ and $\sigma_{2}$ with the property $P_{\sigma_{1}}=P_{\sigma_{2}}$. The dotted curves are integral curves of $j^{\mu}$, on which the arrows indicate the direction of $j^{\mu}$. The arrows on $\sigma_{1}$ and $\sigma_{2}$ indicate the direction of the normal $n^{\mu}$. 
where $s$ is an affine parameter along the curve. (For our purposes, these curves are only an auxiliary mathematical tool, see also [18] for the nonrelativistic case, but we note that in the Bohmian deterministic interpretation such integral curves represent actual particle trajectories [3, 19, 12, 13, 20].) Consider the set of all integral curves that cross the boundary of some spacelike $\sigma_{1}$. This set of integral curves defines a timelike hypersurface. We say that a spacelike $\sigma_{2}$ belongs to the same family of $\sigma^{\prime}$ 's as $\sigma_{1}$ if the set of integral curves above crosses the boundary of $\sigma_{2}$ (see Fig. 11). The Gauss law and Eq. (4) give

$$
\int_{\partial V^{(4)}} d S^{\mu} j_{\mu}=\int_{V^{(4)}} d^{4} x \partial^{\mu} j_{\mu}=0
$$

Since $d S^{\mu}$ is orthogonal to $j^{\mu}$ on the timelike hypersurface defined by the integral curves, (11) implies $P_{\sigma_{1}}=P_{\sigma_{2}}$.

The construction above is consistent for the case in which $j^{\mu}$ is timelike and futureoriented. Indeed, if $\psi(x)$ is a plane wave $\propto e^{-i k_{\mu} x^{\mu}}$ with a positive frequency $k^{0}=$ $\left(\mathbf{k}^{2}+m^{2}\right)^{1 / 2}$, then (2) is timelike and future-oriented. However, for a more general linear combination of plane waves with different positive frequencies, $j^{\mu}$ may not be timelike and future-oriented on some regions of spacetime. The generalization of the construction above to such a general case is the main aim of this paper. The basic idea is to consider hypersurfaces $\Sigma$ that may not be spacelike everywhere. Instead, one can consider hypersurfaces that are spacelike, null, or timelike on the regions on which $j^{\mu}$ is timelike, null, or spacelike, respectively. Can probability density be consistently defined on such regions? For that purpose, consider Fig. 2, which represents an analog of Fig. 1. On the spacelike but past-oriented region $\sigma_{3}$, the timelike vectors $n^{\mu}$ and $j^{\mu}$ have the same direction, so $\tilde{j} \geq 0$, which implies that $\tilde{p}=\tilde{j}$ on $\sigma_{3}$. On the timelike region $\sigma_{2}$ the normal $n^{\mu}$ is spacelike, so the claim that $n^{\mu}$ is "unit" actually means $n^{\mu} n_{\mu}=-1$. The vectors $n^{\mu}$ and $j^{\mu}$ have the same direction, which implies that $\tilde{j} \leq 0$, so it is natural to take $\tilde{p}=-\tilde{j}=|\tilde{j}|$ on $\sigma_{2}$. With these definitions of the probability density, the probability is conserved, i.e., $P_{\sigma_{2}}=P_{\sigma_{3}}$. This can be derived in the same way as for Fig. 1, by using (11) and the fact that $\sigma_{2}$ and $\sigma_{3}$ belong to the same family generated by the same set of integral curves of $j^{\mu}$.

There remains one technical difficulty: how to define $\tilde{p}$ on a null region $\left(\sigma_{1}\right.$ in Fig. 2) ? In particular, how to normalize the normal $n^{\mu}$, i.e., what does it mean that a null vector $n^{\mu}$ is "unit"? To solve this problem, it is instructive to consider a simple example. Let

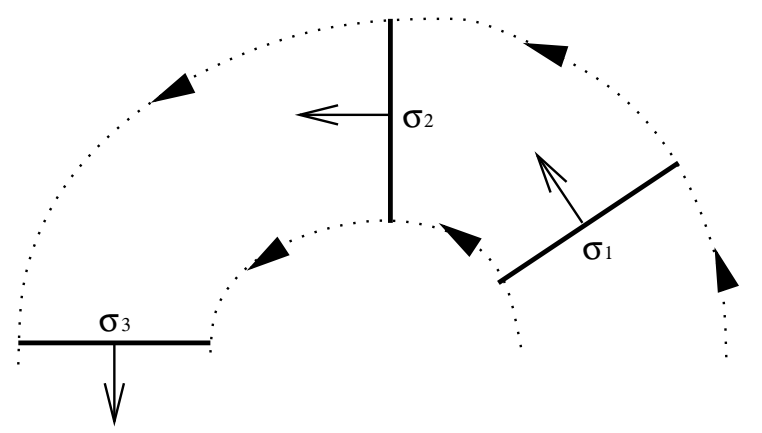

Figure 2: Regions $\sigma_{1}, \sigma_{2}$, and $\sigma_{3}$ with the property $P_{\sigma_{1}}=P_{\sigma_{2}}=P_{\sigma_{3}}$. Here $\sigma_{1}$ is null, $\sigma_{2}$ is timelike, and $\sigma_{3}$ is spacelike but past-oriented. 
$x^{\mu}$ be the standard orthogonal Lorentz coordinates with the Minkowski metric. Consider also the coordinates

$$
x^{\prime 0}=\frac{x^{0}-\beta x^{1}}{\sqrt{2}}, \quad x^{1}=\frac{x^{1}+\beta x^{0}}{\sqrt{2}},
$$

where $\beta$ is a real constant. The coordinates $x^{\prime 0}$ and $x^{11}$ also represent two independent coordinate axes. The hypersurface orthogonal to the axis $x^{\prime 0}$ is spacelike for $|\beta|<1$, null for $|\beta|=1$, and timelike for $|\beta|>1$. The normal to this hypersurface oriented in the direction of the axis $x^{\prime 0}$ is

$$
n^{\mu}=\frac{1}{\sqrt{\left|1-\beta^{2}\right|}}(1,-\beta, 0,0) .
$$

Its norm is

$$
n^{\mu} n_{\mu}=\operatorname{sign}\left(1-\beta^{2}\right),
$$

for $|\beta| \neq 1$. It is convenient to choose the coordinates $x^{\prime 1}, x^{2}$, and $x^{3}$ as the coordinates on the hypersurface orthogonal to the axis $x^{\prime 0}$. From (12), one finds $g_{11}^{\prime}=-2\left(1-\beta^{2}\right) /(1+$ $\left.\beta^{2}\right)^{2}$, so the induced metric on this hypersurface has the property

$$
\left|g^{(3)}\right|^{1 / 2}=\frac{\sqrt{2\left|1-\beta^{2}\right|}}{1+\beta^{2}} .
$$

We see that the case $|\beta| \rightarrow 1$ is singular. In particular, the components of (13) become infinite, while the quantity (15) becomes zero. However, this is only an apparent singularity, because the really relevant quantity in (9) is neither $\left|g^{(3)}\right|^{1 / 2}$ nor $n^{\mu}$, but rather their product

$$
\tilde{n}^{\mu}=\left|g^{(3)}\right|^{1 / 2} n^{\mu} .
$$

From (13) and (15) we find

$$
\tilde{n}^{\mu}=\frac{\sqrt{2}}{1+\beta^{2}}(1,-\beta, 0,0) .
$$

This demonstrates the general rule that $\tilde{n}^{\mu}$ is well defined on all kinds of hypersurfaces, including the null ones.

The results above can be summarized as follows. For arbitrary $j^{\mu}$, one considers hypersurfaces on which the normal $n^{\mu}$ is timelike, null, or spacelike on the regions on which $j^{\mu}$ is timelike, null, or spacelike, respectively. On such hypersurfaces, the probability density is given by

$$
\tilde{p}=\left|\tilde{n}^{\mu} j_{\mu}\right| .
$$

However, even more general foliation of spacetime is admissible. The most general foliation that provides the conservation of probability is the foliation for which each integral curve of $j^{\mu}$ crosses each hypersurface of the foliation once and only once. Equations (7), (11), and (18) imply that, on any such $\Sigma$,

$$
\int_{\Sigma} d^{3} x \tilde{p}=1 .
$$

In addition, for any $\sigma \subset \Sigma$, the probability

$$
P_{\sigma}=\int_{\sigma} d^{3} x \tilde{p}
$$


is invariant, i.e., does not depend on the choice of coordinates on $\sigma$. Note that, for $j^{\mu}$ as in Fig. 2, it is impossible to find an admissible foliation with hypersurfaces that are spacelike everywhere. An example of an admissible foliation is sketched in Fig. 3 ,

\section{Generalization to the many-particle case}

Let us also generalize the results above to many-particle states. For example, the $n$ particle generalization of (3) is [2, 12 ]

$$
\psi\left(x_{1}, \ldots, x_{n}\right)=(n !)^{-1 / 2} S_{\left\{x_{a}\right\}}\left\langle 0\left|\hat{\phi}\left(x_{1}\right) \cdots \hat{\phi}\left(x_{n}\right)\right| n\right\rangle,
$$

where the symbol $S_{\left\{x_{a}\right\}}(a=1, \ldots, n)$ denotes the symmetrization over all $x_{a}$, which is needed because the field operators do not commute for nonequal times. This $n$-particle wave function satisfies $n$ Klein-Gordon equations (11), one for each $x_{a}$. The $n$-particle current generalizing (2) is

$$
j_{\mu_{1} \ldots \mu_{n}}\left(x_{1}, \ldots, x_{n}\right)=i^{n} \psi^{*} \stackrel{\leftrightarrow}{\partial}_{\mu_{1}} \ldots \stackrel{\leftrightarrow}{\partial}_{\mu_{n}} \psi
$$

where $\partial_{\mu_{a}} \equiv \partial / \partial x_{a}^{\mu_{a}}$. It transforms as an $n$-vector [21] and satisfies the conservation equation $\partial_{\mu_{1}} j^{\mu_{1} \ldots \mu_{n}}=0$ and similar conservation equations with other $\partial_{\mu_{a}}$. The generalization of (17) is

$$
\int_{\Sigma_{1}} d S_{1}^{\mu_{1}} \cdots \int_{\Sigma_{n}} d S_{n}^{\mu_{n}} j_{\mu_{1} \ldots \mu_{n}}=1
$$

which does not depend on the choice of timelike hypersurfaces $\Sigma_{1}, \ldots, \Sigma_{n}$. One can also introduce $n$ 1-particle currents $j_{\mu_{a}}\left(x_{a}\right)$ by omitting the integration over $d S_{a}^{\mu_{a}}$ in (23). For example, for $a=1$,

$$
j_{\mu_{1}}\left(x_{1}\right)=\int_{\Sigma_{2}} d S_{2}^{\mu_{2}} \cdots \int_{\Sigma_{n}} d S_{n}^{\mu_{n}} j_{\mu_{1} \ldots \mu_{n}}\left(x_{1}, \ldots, x_{n}\right),
$$

which does not depend on the choice of timelike hypersurfaces $\Sigma_{2}, \ldots, \Sigma_{n}$ and satisfies $\partial_{\mu_{1}} j^{\mu_{1}}=0$. The wave function defined as in (21) provides that different particles cannot be distinguished, which implies that $j^{\mu_{a}}(x)$ does not really depend on $a$. However, for a

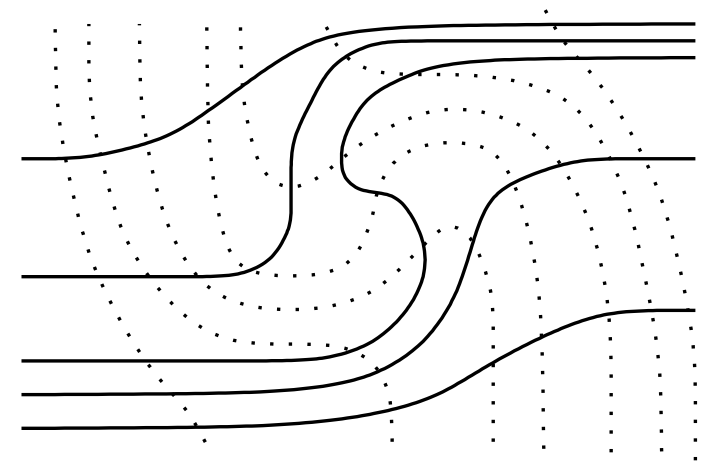

Figure 3: A foliation (solid curves) of spacetime induced by the integral curves (dotted curves) of $j^{\mu}$. 
more general $n$-particle wave function, $j^{\mu_{a}}(x)$ may depend on $a$. The integral curves of $j^{\mu_{a}}\left(x_{a}\right)$ determine admissible foliations. The "unit" normal on a hypersurface of such a foliation is $n^{\mu_{a}}\left(x_{a}\right)$, while the determinant of the induced metric on this hypersurface is $g_{a}^{(3)}\left(x_{a}\right)$. Introducing

$$
\tilde{n}^{\mu_{a}}\left(x_{a}\right)=\left|g_{a}^{(3)}\left(x_{a}\right)\right|^{1 / 2} n^{\mu_{a}}\left(x_{a}\right),
$$

the probability density generalizing (18) is

$$
\tilde{p}\left(x_{1}, \ldots, x_{n}\right)=\left|\tilde{n}^{\mu_{1}}\left(x_{1}\right) \cdots \tilde{n}^{\mu_{n}}\left(x_{n}\right) j_{\mu_{1} \ldots \mu_{n}}\left(x_{1}, \ldots, x_{n}\right)\right| .
$$

(Note that a construction similar to (26) is discussed in [19] for the case of fermions. However, in the case of fermions, it is not necessary to introduce hypersurfaces that are not spacelike everywhere.) The probability of finding one particle on $\sigma_{1}$, another particle on $\sigma_{2}$, etc., is

$$
P_{\sigma_{1}, \ldots, \sigma_{n}}=\int_{\sigma_{1}} d^{3} x_{1} \cdots \int_{\sigma_{n}} d^{3} x_{n} \tilde{p}\left(x_{1}, \ldots, x_{n}\right) .
$$

This is a variant of the many-time probability $P\left(t_{1}, \ldots, t_{n}\right)$ [22], modified such that the regions $\sigma_{a}$ orthogonal to $n^{\mu_{a}}$ may not be spacelike.

\section{Discussion and summary}

Now let us discuss the issue of causality related to the foliation of spacetime with hypersurfaces that are not spacelike everywhere. One might think that such a foliation could be related to particles that can move faster than light or backwards in time. Indeed, in the Bohmian deterministic hidden-variable interpretation, such motions are possible [19, 12, 20]. However, the deterministic evolution of the wave function $\psi$ is, of course, causal, irrespective of the probabilistic interpretation of $\psi$. Consequently, with the conventional purely probabilistic interpretation on hypersurfaces that are not spacelike everywhere, one cannot use the foliation with such hypersurfaces to send information to the past or faster than light. In this sense, causality is not violated.

Finally, let us make a few remarks on the problem of measurement. We observe that, in nonrelativistic QM, it is not so trivial to predict a probability density such as $p(t, x, y)$ for a fixed $z$, despite the fact that such a probability density can be determined experimentally. The problem is that the corresponding measurements cannot be attributed to only one equal-time hypersurface. Consequently, to make predictions on such measurements, one must deal with a theory of quantum measurements that involves the problematic concept of "wave-function collapse" or some substitute for it. Analogous problems occur in our relativistic theory as well, when one wants to make predictions on measurements that cannot be attributed to only one admissible hypersurface. The solutions of such problems are expected to be analogous to those in nonrelativistic QM, but a detailed discussion of these aspects is beyond the scope of the present paper.

To summarize, in this paper we have shown that the conserved currents associated with relativistic wave equations can be consistently interpreted as probability currents. However, the main novel feature is that the shape of hypersurfaces on which the probability density can be defined depends on the direction of the current. In other words, the wave function (from which the current is calculated) determines not only the probability 
density, but also the admissible hypersurfaces on which this probability density is defined. A curious but consistent feature of these hypersurfaces is that they may not be spacelike everywhere.

\section{Acknowledgments}

The author is grateful to D. Dürr, S. Goldstein, and R. Tumulka for valuable discussions. This work was supported by the Ministry of Science and Technology of the Republic of Croatia.

\section{References}

[1] J. D. Bjorken and S. D. Drell, Relativistic Quantum Mechanics (McGraw-Hill, New York, 1964).

[2] S. S. Schweber, An Introduction to Relativistic Quantum Field Theory (Harper \& Row, New York, 1961).

[3] K. Berndl, D. Dürr, S. Goldstein and N. Zanghì, Phys. Rev. A53, 2062 (1996).

[4] J. D. Bjorken and S. D. Drell, Relativistic Quantum Fields (McGraw-Hill, New York, 1965).

[5] H. Nikolić, quant-ph/0609163, to appear in Found. Phys.

[6] T. D. Newton and E. P. Wigner, Rev. Mod. Phys. 21, 400 (1949).

[7] T. O. Philips, Phys. Rev. 136, B893 (1964).

[8] A. S. Wightman and S.S. Schweber, Phys. Rev. 98, 812 (1955).

[9] H. Nikolić, Phys. Lett. B527, 119 (2002).

[10] H. Nikolić, Int. J. Mod. Phys. D12, 407 (2003).

[11] H. Nikolić, Gen. Rel. Grav. 37, 297 (2005).

[12] H. Nikolić, Found. Phys. Lett. 17, 363 (2004).

[13] H. Nikolić, Found. Phys. Lett. 18, 123 (2005).

[14] R. Oeckl, Phys. Lett. B575, 318 (2003).

[15] C. Rovelli, Quantum Gravity (Cambridge University Press, Cambridge, 2004).

[16] L. Doplicher, Phys. Rev. D70, 064037 (2004).

[17] H. Nikolić, Eur. Phys. J. C42, 365 (2005).

[18] C. L. Lopreore and R. E. Wyatt, Phys. Rev. Lett. 82, 5190 (1999). 
[19] D. Dürr, S. Goldstein, K. Münch-Berndl and N. Zanghì, Phys. Rev. A60, 2729 (1999).

[20] H. Nikolić, Found. Phys. Lett. 18, 549 (2005).

[21] B. S. DeWitt and R. W. Brehme, Ann. Phys. 9, 220 (1960).

[22] S. Tomonaga, Prog. Theor. Phys. 1, 27 (1946). 